\title{
OBSERVATIONS ON INTERNATIONAL COOPERATION
}

\author{
G. WOLF \\ Head of Fusion Project Management, Kfa, Julich
}

\begin{abstract}
Dr Pease, I might rather say Bas, Ladies and Gentlemen,
\end{abstract}

As A YouNG plasma physicist twenty years ago I had the opportunity of working with you here at the Culham Laboratory while on one-year leave from Max Planck Institute of Plasma Physics in Garching. This was caused by a common scientific interest namely questions of the stability of a toroidal high- $\beta$ configuration. My time here at Culham meant a great deal to me both personally and professionally. It was an exciting and decisive phase in fusion research, an intersection of ideas and results, a kind of abstract of what you have already heard today.

The experiments with the unique $8 \mathrm{~m}$ theta pinch showed amongst other results, that a bumpy configuration is magnetohydrodynamically unstable. The CLASP experiment demonstrated that electrons can be confined in the magnetic field of a stellarator for at least $10^{7}$ toroidal revolutions. Shortly before its shutdown, the famous ZETA experiment gave a surprising result namely the 'quiescent phase', which revealed a new class of toroidal confinement called the reversed field pinch. The Culham expedition to participate in the tokamak experiments in Moscow followed soon after this, and the tokamak's rise began, of which JET here in Culham is the most impressive example.

Stimulated by discussions with Gerhard Berge, Hugh Bodin, John Wesson and in particular with Bas Pease, I started to think about the Rayleigh-Taylor instability, which later benefited me in my own experiments on dynamic stabilization.

Visitors such as Ellis, Griem, Keilhacker, Killeen, Malesani, Segre and Uchida were attracted to Culham immediately before and after my visit. This was a time of lively scientific arguments in an atmosphere of friendship. The reception I enjoyed at that time still makes me feel in a certain sense at home here in Culham even today. My experience as a young visiting scientist illustrates the first and perhaps the most important reason for international cooperation, namely understanding, respect and friendship between scientists from various nations and peoples.

In the following, however, I shall not speak so much about this general feature. Instead, I shall try to follow the invitation to present some thoughts on more specific aspects of international cooperation, including the experience from my own field of work. It gives me particular pleasure to talk about this topic on the present occasion because Bas Pease has contributed so much to this subject: it is a subject close to the heart.

I would first like to draw attention to a trivial fact international cooperation does not a priori mean a multiplication of the available resources but rather only a different method 
of applying them. Various factors are involved in deciding whether international cooperation results in an increase or reduction of the overall potential of the partners.

At least on the part of governments, pressure towards increased scientific cooperation often only conceals the understandable desire to keep their own contribution as small as possible. This is frequently put forward as the intention of avoiding duplication but fails to recognize the fact that to a certain extent duplication and reproducibility are necessary prerequisites for scientific and technical progress in the same way as pluralism of ideas, competition and scientific independence. However, governments appear to favour international cooperation due to a further motive, particularly with respect to the planning and implementation of joint large-scale projects such as INTOR or more recently ITER, namely their symbolic significance for a future free of international conflicts and removed from present day problems.

What then are our own motives for scientific cooperation in fusion research where our path towards the goal is still long, laborious and expensive, and where very large experimental facilities are required? Although already the unimpeded communication of results is sometimes considered as a kind of cooperation, what I will address in the following is aimed more at actively working together on joint tasks. This also includes of course, the exchange and accumulation of ideas, plans, computer codes such as the Princeton TRANSP-Code, blueprints and equipment. From what I can see, there are six reasons for engaging in such activities:

(1) To build and operate devices, which in size, cost and numbers of professionals would exceed the efforts a single nation is able or willing to invest.

(2) To bring together experts and equipment from all over the world to work jointly on a special task and at the same time to provide a stimulating and creative atmosphere; this also means to establish centres for one particular task in the sense of a worldwide division of labour.

(3) To integrate the national research programmes of smaller nations into a larger framework and a coordinated effort, yet still maintaining and strengthening their own identity and their chance to participate in the expected future benefits.

(4) To maintain a sufficiently broad spectrum of research determined by the present state of the art, or putting it another way, to prevent the programmes being narrowed at too early a stage as may result from too optimistic planning or from just jumping on the bandwagon.

(5) To obtain some political stabilization of our long-term enterprise in view of changing governments and of changing political priorities and trends.

(6) To become aware of a common worldwide task and to create an international scientific community directed towards these common goals.

It is evident from Prof. Palumbo's speech that these aspects have been realized in the fusion programme of the European Community in an excellent manner. Therefore, we tend to use the concept of 'international cooperation' less and less to describe the cooperation within the European Community, since this has already become a matter of course for us. Instead, we now reserve the expression 'international cooperation' mainly to characterize joint work between the EEC and other major industrial nations such as Japan, the USA and USSR. Even twenty years ago we would never have imagined that such a rapid and extensive integration of the European fusion programme would be possible, an integration which has enabled us to achieve a leading position in the world, par- 
ticularly with JET. This is a debt we owe to the European Community.

At this point, Ladies and Gentlemen, I would like to make a few personal comments on two quite different subjects, namely the European Community on the one hand and JET on the other.

As a member of a generation who experienced the Second World war and its consequences, I regard the European Community as one of our most important political achievements which cannot be valued too highly. Therefore, I am concerned about possible repercussions of the following opinion repetitively expressed by our media, namely that there is a lack of radical solutions to ongoing problems which is caused by the slow pace of our European institutions.

From my point of view, these criticisms ignore or misunderstand many aspects. Firstly, that we have achieved in Europe a degree of personal freedom, social security and standard of living never experienced before. Secondly, that agricultural overproduction-the most frequently mentioned problem in the media-is most certainly better than its converse, namely famine. Thirdly, it is frequently forgotten that the European Community was not created to solve the existing problems at one stroke but rather to establish the procedures to deal with these problems in a peaceful and equitable manner. It is quite natural and indeed unavoidable that problems should exist between nations and individuals: what counts is the way in which we deal with these problems and how we attempt to solve them. Finally, by focusing exclusively on the solution of traditional problems and on the structural integration of different countries these criticisms neglect the importance of future oriented joint undertakings which can indeed only be tackled by an institution such as the European Community. An example of this is the fusion reactor.

Which brings me back to fusion research and JET, the subject of my second personal remark. I am not aware of any important decision concerning JET which-even with hindsight-may be considered wrong. That means we have chosen the right machine, the right people, the right programme, and naturally have also had the right amount of luck. Of course, this does not lead us to rest upon our laurels, instead it emphasizes the importance of forthcoming decisions for the future of JET and the JET team so that we may continue to deal wisely with this our most important asset, that is to say with the queen in our present game of chess.

I would like now to discuss a few aspects from my own field of experience in connection with international cooperation. As you all know, our research programme on TEXTOR in Julich is characterized by the fact that we have established close cooperation agreements both within Europe and on an intercontinental basis.

Within the INTER-EUROPEAN dimension, emphasis must be placed on our partnership with the EURATOM-Belgian State Association which-dispensing with their own possible large tokamak-have completely integrated their independent research programme 'High-Frequency Heating of Plasmas' into the Julich tokamak programme and thus given a significant additional dimension to the TEXTOR programme. Paul Vandenplas and his team of scientists have now become friends, equal partners and colleagues on TEXTOR, and they participate fully in the internal decision-making procedures.

Furthermore, we have also recently agreed upon a joint project with our other European next door neighbours, namely with the FOM Institute at Rijnhuizen. This con- 
cerns the application of Rutherford scattering to measure ion temperature on the basis of extensive development work carried our by our Dutch colleagues.

Our international cooperation is regulated by an agreement, within the framework of the International Energy Agency formally granting the partner countries of Canada, Japan, Switzerland and the USA the right to make use of $40 \%$ of TEXTOR's operating time for their own research goals in the field of plasma-wall interaction. This formal regulation has in practice led to joint programmes which are carried out mostly by joint, integrated groups consisting both of scientists and technicians from the partner countries as well as KFA staff.

The most recent and to date most significant project within the framework of the IEA Agreement has been the joint construction and operation of a toroidal pump limiter system. It was designed, constructed and financed on a joint basis by three of the partners and is now an integral component of TEXTOR. Nevertheless, the satisfactory conclusion of the construction phase of this project should not distract attention from the fact that apart from the advantages already mentioned, international cooperation also involves considerable efforts and indeed risks which may only be kept under control with the tolerance and good will of all partners. To my mind, it is not enough to negotiate ever more sophisticated legal clauses as a precaution against these possible risks. On the contrary this can lead to grotesque delays in concluding the agreement. Rather the partners must be tacitly or explicitly prepared to take over the agreed obligations of a contracting party if he is unable to perform them, or else be prepared to reduce the scope of the project.

Let me take up the example of the toroidal pump limiter on TEXTOR once again in order to illustrate some of the procedures and indeed difficulties involved in intercontinental cooperation. The first step is the expression of intent by the scientific representatives of the partners, i.e. in our case amongst the members of the IEA TEXTOR Executive Committee and their advisors. This includes defining the scientific goals the technical design of the facility and then the optimum division of labour between the partners, and also the diagnostics, data acquisition, data link etc. An international design team must be established and attention must be paid to the various interfaces. This means that the work and coordination required as a matter of course in setting up a complicated project has to be carried out jointly by the partners in three continents, and the fact that it has really worked should not conceal the considerable efforts behind it.

Furthermore, this means that not only one approval procedure will be required, as otherwise usual, but rather as many as the number of partners involved each with their different criteria, practices, constraints, and fiscal years. Each of these licensing procedures will then require its own scientific workshop and its committee of experts, occasionally involving several stages. Failing just one of these parallel steps could jeopardise the entire project. It thus becomes clear that only the willingness to accept risks and a great deal of good will on the part of all those involved can lead to a successful conclusion.

Finally, it must not be forgotten that apart from these procedures there were and indeed still are serious technical problems. In other words, the procedure is not an end in itself but rather serves a complicated technical goal. Therefore, in future we should attempt in similar cases to reduce or simplify the number of approval procedures running parallel, which would be a task for discussions between the appropriate authorities. 
Last but not least, we still need practice in constructing complex apparatus and in implementing programmes with teams scattered throughout the world, and this educational process must also pay attention to an understanding of individual national characteristics.

Let us now turn form this example and look further into the future. We know quite well that in international projects compromise will become inherently unavoidable. These compromises may involve the schedule, the distribution of work and costs and many other aspects. Nevertheless, they should not lead to an impairment of those qualities of a joint project which determine the chances of final scientific success. This means that we should not accept any compromises with respect to the qualification of the scientists to be selected nor with respect to the definition, design and implementation of the machine as such. In fact, to get involved in the tedious procedure of a really large-scale international project may be only worthwhile at all if we do not compromise on its main potential benefit which is to obtain both the budget and the staff required for building and exploiting an outstanding machine.

Obviously, these principles and the hard facts of life may contradict each other to some extent. Therefore, it is important to select a strong and competent project management team with one director, and to choose the right balance in the interdependence between that team and the international supervisory committees which by nature will be more exposed to the demands for compromise. Furthermore, a buffer mechanism is needed to protect the continuity and development of the project during its entire lifetime of for example 20 years from conceivable ups and downs in the attitude of individual partners. Novel ideas differing from the general opinion should also receive a fair chance. In addition, the project should be so attractive that top scientists clamour to take part in it.

In this sense it seems to me that we Europeans, due to our special situation, have developed the attitude and the procedures for dealing with these problems and that we are doing the right thing in transferring our experience with JET and more recently with NET to large intercontinental projects such as ITER. I think we should congratulate all colleagues involved in the negotiations on ITER on having successfully achieved the first steps in the right direction. In this context, one might consider whether some of the procedures which nations have developed for their diplomatic services to work in foreign countries, should not be also applied to such future international projects and their teams. This might be helpful not only in a general sense but perhaps also alleviate the difficult problems of agreeing on a specific site.

I have tried to present a few observations and ideas concerning international cooperations and I would like to summarize my remarks as follows.

International cooperation is an excellent catalyst for respect and tolerance between nations. Since international cooperation makes it possible in principle to work on largescale and long-term goals, controlled nuclear fusion is a particularly suitable subject for such an undertaking. However, this means that all partners must seriously and persistently pursue this aim and that mechanisms have to be developed to buffer fluctuations in budgetary conditions and in political fashions and trends in order to accommodate the long-term nature of the goal. Let me put it like this, fusion needs international cooperation and fusion is an excelient vehicle for international cooperation.

As we all know, you Bas Pease, were one of the very first to perceive and realize this concept. As chairman of the IAEA International Fusion Research Council, of the Pro- 
gramme Committee of the CCFP and in many other positions, such as on the Committee of Directors you promoted fusion and international cooperation. In this context I should particularly emphasize your continuous engagement in launching and supporting the INTOR study which also served as the predecessor to the recent ITER activity. I would like to express our deep thanks. 